English Language and Linguistics 5.2: 251-272. (C) Cambridge University Press 2001 DOI: $10.1017 / \mathrm{S} 1360674301000235$ Printed in the United Kingdom

\title{
The English present tense
}

\author{
RONALD W. LANGACKER \\ University of California, San Diego \\ (Received 3 November 2000; revised 28 May 2001)
}

\begin{abstract}
It is generally agreed that the English 'present tense' is not appropriately analyzed as indicating present time: present-time events often cannot be expressed in the present tense; conversely, the present tense is often used for nonpresent occurrences. I will argue, however, that these problems are only apparent, arising from a failure to appreciate the numerous conceptual factors that are crucially involved. When these are properly elucidated, using notions available in cognitive semantics and cognitive grammar, the characterization 'coincidence with the time of speaking' proves remarkably adequate in accounting for present-tense usage.
\end{abstract}

\section{The problem}

A characterization of the English present tense should, one would think, be simple and straightforward. What could be more basic than an indication of present time? And what could be simpler morphologically than its typical exponent, namely zero, the absence of any verbal inflection?

Yet the English present is notorious for the descriptive problems it poses. Some would even refer to it as 'the so-called present tense in English', so called because a characterization in terms of present time seems hopelessly unworkable. On the one hand, it typically cannot be used for events occurring at the time of speaking. To describe what I am doing right now, I cannot felicitously use sentence (1a), with the simple present, but have to resort to the progressive, as in (1b).

(1) (a) *I write this paper right now.

(b) I am writing this paper right now.

On the other hand, many uses of the so-called present do not refer to present time at all, but to the future, to the past, or to transcendent situations where time seems irrelevant:

(2) (a) My brother leaves for China next month.

(b) I'm eating dinner last night when the phone rings. I answer it but there's no response. Then I hear this buzzing sound.

(c) The area of a circle equals pi times the square of its radius.

It might appear, in fact, that the present tense can be used for anything but the present time.

I am going to argue, however, that these problems are only apparent, arising from a failure to appreciate all the conceptual factors involved in the various sorts of expressions. Once these factors are properly elucidated, a characterization of the present tense as indicating 'coincidence with the time of speaking' proves remarkably 
adequate in accounting for its usage. I am claiming, in other words, that the socalled present tense does in fact deserve to be so called.

\section{Cognitive grammar}

Before describing the so-called 'so-called present tense', I need to introduce a few basic notions from the theory of cognitive grammar (Langacker, 1987a, 1990, 1991, 1999a). The theory's architecture directly reflects the semiological function of language: that of allowing conceptual structures to be symbolized by sound structures. To fulfill this function, a language must at least comprise semantic structures, phonological structures, and symbolic links between the two. Cognitive grammar claims that only these elements are necessary.

In contrast to standard doctrine emphasizing modularity and the autonomy of syntax, cognitive grammar achieves conceptual unification by recognizing the inherent meaningfulness of grammatical structure. Grammar is claimed to be symbolic in nature. A symbolic structure is defined as the linking of a form (i.e. a phonological structure) and a meaning (a semantic structure). Lexicon, morphology, and syntax make up a continuum consisting solely of assemblies of symbolic structures. Consequently, all elements validly posited in grammatical description have some kind of meaning (often quite schematic).

The reduction of grammar to symbolic assemblies requires an appropriate and independently justified view of semantic structure. Meaning is identified with conceptualization, in the broadest sense of that term (i.e. any kind of mental experience). It thus subsumes all of the following: (i) both established and novel conceptions; (ii) not only abstract or intellectual 'concepts' but also immediate sensory, motor, and emotive experience; (iii) conceptions that are not instantaneous but change or unfold through processing time; and (iv) full apprehension of the physical, social, and linguistic context. Crucially, moreover, we have the ability to construe the same situation in many different ways. Linguistic meanings are a function of both the conceptual content evoked and the construal imposed on that content. As part of its conventionally determined value, every linguistic expression or symbolic structure incorporates a particular way of construing its own conceptual content or that evoked by other elements.

Out of the many aspects of construal, only a few can be mentioned here. First is the level of specificity - or conversely, schematicity - at which a situation is characterized. Thus each expression in (3a) is schematic with respect to the one that follows, which makes more detailed, finer-grained specifications. Another dimension of construal is perspective, which subsumes a number of factors. One factor is a presupposed vantage point, illustrated by the contrast between go and come in (3b). A second factor is the path of mental access followed by the conceptualizer (i.e. the speaker, and secondarily the hearer). In (3c), for instance, the contrasting expressions reflect alternate directions of mental scanning in conceptualizing what is objectively the same situation. 
(a)

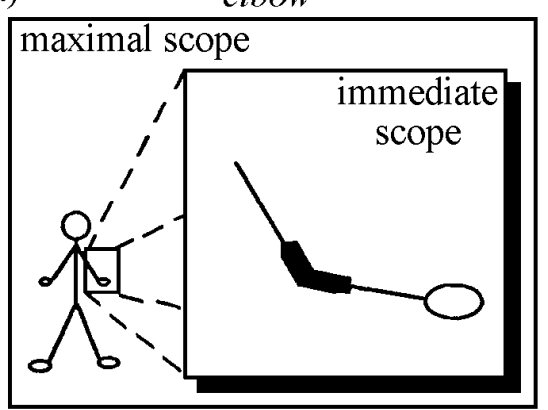

(b)

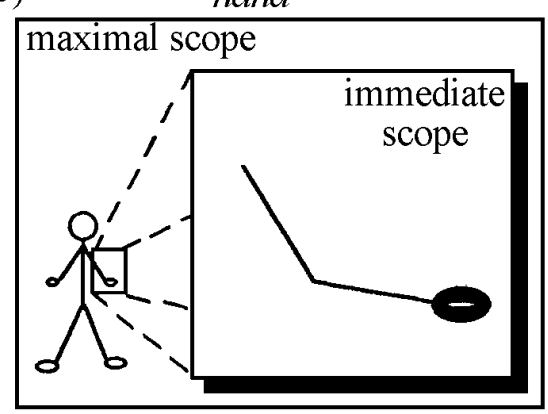

Figure 1. Scope

(3) (a) thing $\rightarrow$ creature $\rightarrow$ animal $\rightarrow$ dog $\rightarrow$ poodle

(b) She $\{$ went/came $\}$ up into the attic.

(c) The scar extends \{from his wrist to his elbow/from his elbow to his wrist\}.

Two additional dimensions of construal are scope and prominence. An expression's scope is defined as the array of conceptual content it evokes as the basis for its meaning. Its maximal scope is the full array of content evoked. Within the maximal scope, there is often a more limited immediate scope comprising the specific array of content directly relevant for a particular purpose or at a particular level of organization. Metaphorically we can think of an expression's immediate scope as the 'onstage region', or general locus of viewing attention. Clear examples of these notions are provided by whole-part hierarchies. While the maximal scope for terms like elbow and hand is the conception of the human body as a whole, as shown in figure 1, it is evident that the arm in particular is most directly relevant to their characterization. The conception of the arm thus functions as their immediate scope, which - being the general locus of attention - is more prominent in these expressions than are other parts of the body.

There is considerable linguistic motivation for adopting the notion immediate scope (Langacker, 1993), which is necessary for both semantic description and the statement of various grammatical patterns. In a whole-part hierarchy, like body $>$ arm $>$ hand $>$ finger $>$ knuckle or body $>$ head $>$ face $>$ eye $>$ pupil, the referent of each expression functions as immediate scope with respect to the next. One grammatical phenomenon sensitive to this hierarchy is the formation of noun-noun compounds designating body parts, such as fingernail, earlobe, eyelid, bellybutton, kneecap, etc. Observe that the first member of the compound designates the immediate scope for the second. Violations of this restriction yield compounds that are strikingly infelicitous: *armnail, *headlobe, *facelid, *bodybutton, *legcap.

As the general locus of attention, an expression's immediate scope is more salient than other portions of its maximal scope. Hence selection as immediate scope represents one kind of prominence, out of the numerous kinds that need to be recognized and distinguished for linguistic purposes. Especially important here is the 
(a) Conceptual Base

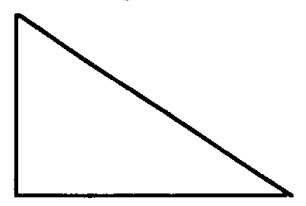

\section{hypotenuse}

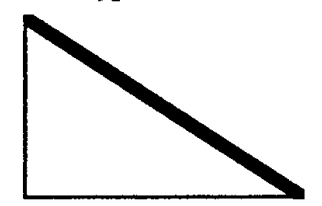

(b) Conceptual Base
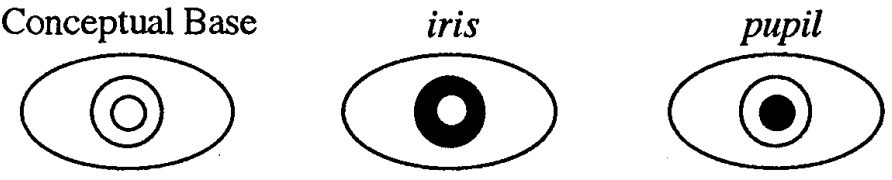

(c)
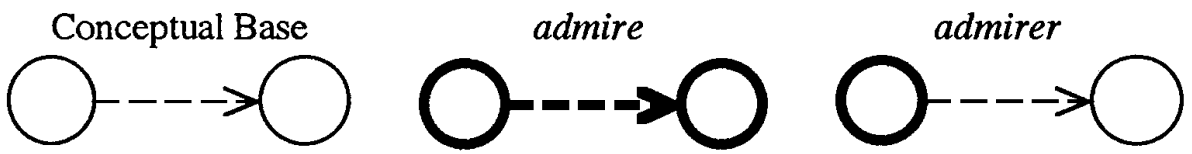

Figure 2. Profiling

type of prominence called profiling (indicated diagrammatically by means of heavy lines). Within its conceptual base (the content it evokes), every expression singles out a particular substructure as its profile. This is the entity it designates, i.e. its conceptual referent. The profile is also describable as the specific focus of attention within the expression's immediate scope. Often two expressions have the same conceptual base but differ semantically owing to their choice of profile. Observe in figure 1 that elbow and hand impose different profiles on the same immediate scope in space.

Further examples are given in figure 2. The term hypotenuse evokes as its base the conception of a right triangle, within which it profiles the side opposite the right angle. Iris and pupil impose different profiles on the base comprising the overall configuration of an eye. In figure 2(c), the dashed arrow represents a particular kind of mental attitude which a sentient creature holds with respect to some other entity. The verb admire profiles this mental relationship, whereas the derived noun admirer evokes it as its base but profiles only the individual who entertains the attitude.

A basic claim of cognitive grammar is that an expression's grammatical class is determined by the nature of its profile, not by its overall content (Langacker, 1987a: chs. 5-7, 1987b). A noun profiles a thing (abstractly defined). A verb profiles a process, characterized as a relationship scanned sequentially in its evolution through time. Various other classes (e.g. adjective, adverb, preposition, participle) profile relationships that are nonprocessual. Despite their identical content, admire is thus a verb because it profiles a mental relationship (viewed in its evolution or persistence through time), while admirer is a noun because it profiles a kind of thing.

Further exemplification is given in figure 3. Like any verb, perform involves the 

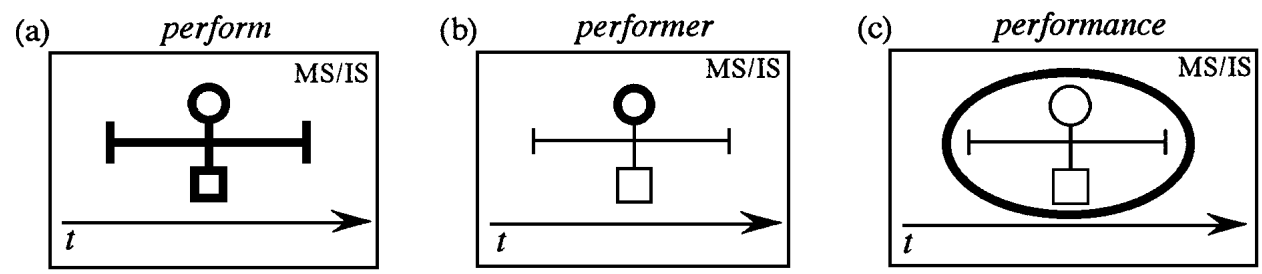

Figure 3. Profiling and grammatical category

domain of time (the arrow labeled $t$ ) and profiles a relationship followed in its evolution along this axis. At this level there is no reason to distinguish between the maximal scope (MS) and the immediate scope (IS) in the temporal domain, hence they coincide (MS/IS). ${ }^{1}$ A vertical line represents the profiled relationship, and the circle its central participant. The horizontal bar stands for the evolution of this relationship through time, with endpoints indicated to specify that the designated process is construed as being bounded. The derived forms performer and performance evoke this process as their conceptual base, hence they have the same content, but since they profile things (rather than the process per se), they are categorized as nouns. Like admirer, performer profiles the central relational participant. On the other hand, performance derives by conceptual reification of the designated process, creating an abstract thing corresponding to the event as a whole. It is this abstract entity (shown as an ellipse) that is profiled (Langacker, 1991: 1.2).

\section{Perfective and imperfective verbs}

It is well known that English verbs divide into two basic aspectual classes on the basis of their grammatical behavior. My own terms for these classes are perfective and imperfective. The usual diagnostics for the classification are occurrence in the simple present tense (with actual present-time meaning) and occurrence in the progressive. Perfectives resist the simple present but take the progressive, while imperfectives do the opposite:

(4) (a) *He builds a house.

( $\left.\mathrm{a}^{\prime}\right) \mathrm{He}$ is building a house. [perfective] (b) He knows the truth. $\quad\left(b^{\prime}\right) * H e$ is knowing the truth. [imperfective]
[ime

Elsewhere (1987a, 1987b) I have argued in detail that the perfective/imperfective distinction is exactly equivalent to the count/mass distinction for nouns, once the intrinsic nature of nouns and verbs is taken into account. The defining feature of count vs. mass nouns is whether or not the noun's referent, i.e. the thing it profiles, is construed as being bounded. Because an expression's grammatical class is determined by the nature of its profile, and the profile by definition is the focus within its immediate scope (the general locus of attention), the bounding in question must

1 This is analogous to a syllable coinciding with a word, or a clause with a full sentence. 
(a)

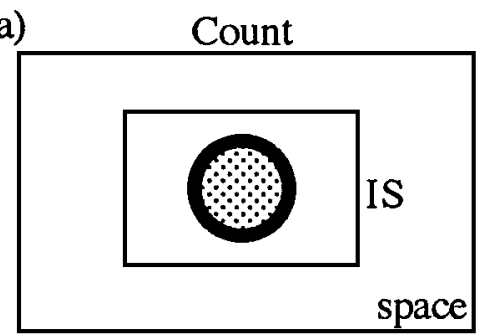

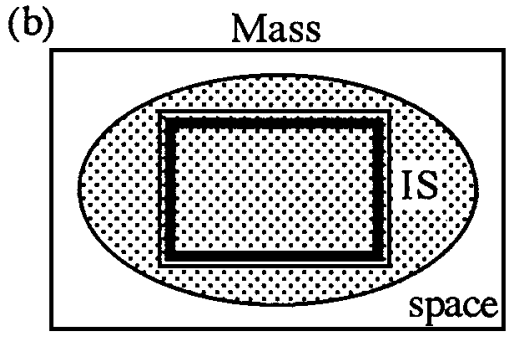

Figure 4. Noun classes

occur within the immediate scope to be relevant. Concentrating on physical objects and substances, where the domain that matters for this purpose is space, we can describe the distinction as follows: a count noun profiles a thing construed as being bounded within the immediate spatial scope, whereas a mass noun profiles a thing not specifically construed as being bounded.

This distinction is diagrammed in figure 4 , where shading indicates the substance constituting the profiled entity. With a count noun, the boundaries of the profiled thing fall within the immediate scope in space. By contrast, the overall expanse of substance designated by a mass noun may well overflow the limits of the immediate spatial scope, as shown in 4(b). In this case, the nominal profile includes only that portion of the mass subtended by the immediate scope, since an expression's profile is the onstage focus of attention and is thus confined to the immediate scope as a matter of definition.

Let us consider just a single example. With the verb see, the limits of the visual field define the immediate scope for purposes of characterizing the direct object. Granted that the description is solely based on the speaker's current visual experience (excluding inference and prior knowledge), we thus observe the contrast in (5) with respect to the same body of water. Sentence (5a) presupposes a global view such that the boundary falls within the visual field, as in figure 4(a). In this case the count noun lake is used to describe the body of water in question. On the other hand, (5b) suggests a local view in which the boundary is not visible, as depicted in figure 4(b). Since the referent is unbounded within the immediate spatial scope (defined by the visual field), the mass noun water is used instead.

(5) (a) I see a lake. [viewed from a plane; boundaries apparent; count noun] (b) I see (a lot of) water. [while swimming; boundaries not visible; mass noun]

One additional pertinent fact concerning count and mass nouns is that any subpart of a mass is itself a valid instance of the mass-noun category. Thus, given an instance of the water category (e.g. a liter), a subpart (even a single drop) is also an instance of water. The same is not in general true for count nouns: part of a lake is not itself a lake, nor does the tail of a cat qualify as a cat. This difference follows from the description of the two basic classes. Because a mass is construed as being internally homogeneous, and bounding does not figure in its characterization, any 
(a)

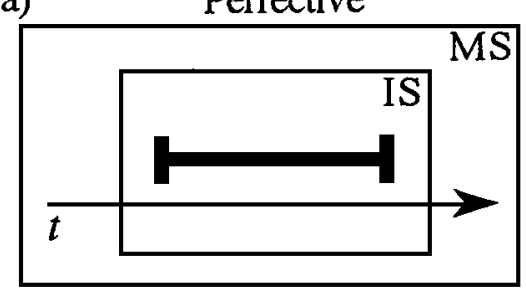

(b) Imperfective

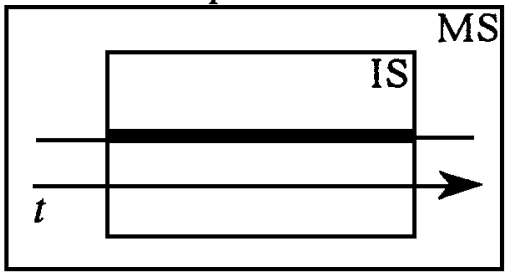

Figure 5. Verb classes

subpart of a mass is itself a valid instance of the mass-noun category. But since a count noun refers to a bounded whole, an arbitrary limited portion of an instance does not itself constitute a valid instance of the count-noun category.

Let me now draw the analogy to perfective and imperfective verbs. Instead of a thing, a verb profiles a process, i.e. a relationship scanned sequentially in its evolution through time. For verbs, consequently, the domain of relevance is always time (as opposed to space). The perfective/imperfective contrast thus hinges on whether the profiled process is construed as being bounded within the immediate scope in the temporal domain: a perfective verb profiles a process construed as being bounded within the immediate temporal scope, whereas an imperfective verb profiles a process not specifically construed as being bounded.

The diagrams in figure 5 are thus the processual analogs of those in figure $4 .^{2} \mathrm{~A}$ perfective process is bounded within the immediate temporal scope. An imperfective process is a constant situation persisting indefinitely through time. However, only that portion which falls within the immediate temporal scope constitutes the processual profile, because an expression's profile is by definition confined to the onstage region.

The restricted profiling shown in figure 5(b) does not imply that the situation itself is limited to the immediate scope. To the contrary, some portion of a continuing situation is being selected for focused viewing. The immediate scope is the general locus of attention, in this case the span of time being attended to. Hence the profiling of an imperfective process usually involves a kind of sampling, where only a portion of an ongoing situation is selected for examination. In (6), for instance, the period chosen for focused viewing is the duration of the interview. It is not implied that the profiled process (that of her being relaxed) began at the onset of the interview or stopped when it ended. The import is rather that the situation in question was in effect and constant throughout the interval examined.

(6) During the interview she was quite relaxed.

We have seen that any subpart of a mass is itself a valid instance of the mass-noun

2 Since only temporal factors are relevant here, I have suppressed the vertical line used in figure 3 to represent the profiled relationship itself. For present purposes we need only examine the horizontal bar indicating its evolution through time. 
category. Given the analogy between mass nouns and imperfective verbs, we should likewise expect any subpart of an imperfective process to itself be a valid instance of the imperfective process category. It is in fact this property of imperfectives which permits the sampling illustrated in (6) and figure 5(b): when the profile is limited to the portion of an imperfective situation subtended by the immediate temporal scope, that portion itself instantiates the imperfective process type. This property follows from the characterization of imperfectives as being unbounded and internally homogeneous. It does not of course hold for perfectives, the analog of count nouns. Since a perfective process is construed as a bounded whole, an arbitrary limited portion of an instance does not itself constitute a valid instance of the perfective verb category. Consider just one example: the imperfective process know a poem, versus the perfective process learn a poem. If I know a poem for a month or more, then for any interval one cares to examine within that period - a week, a day, or an hour - it can also validly be said that $I$ know it. But if it takes me a month to learn a poem, what happened during a shorter period, e.g. a week, cannot be described by saying that I learned it.

Some additional observations need to be made about count and mass nouns, on the one hand, and perfective and imperfective verbs, on the other. First, there are cases of both count nouns and perfective verbs whose referents are internally homogeneous, hence mass-like, but are nonetheless construed as being bounded. This bounding is sufficient to effect their categorization as count or perfective, despite their internal homogeneity. For example, the count nouns lake, lawn, [a] steak, and [a] rock are respectively comparable to the mass nouns water, grass, steak, and rock, except that the count noun specifically profiles a bounded expanse of the substance in question. As a mass noun, for instance, steak merely refers to a kind of meat, but as a count noun it specifically designates one bounded slab of this substance. Likewise, the following verbs are readily construed as being internally homogeneous, yet in English they clearly function as perfectives, according to the usual diagnostics (e.g. they can all take the progressive): sleep, dream, run, walk, sit, stand, lie, perspire, talk, chat, meditate, wear a tie. I suggest that such processes are construed as occurring in bounded episodes, and that this is part of the conventionalized meanings of these verbs in their basic senses.

This is however a matter of how particular situations are construed, which cannot always be determined from their objectively discernible properties. We can see this quite clearly with posture verbs - sit, stand, and lie - which can function as either perfectives or imperfectives. Consider these examples:

(7) (a) Fred is lying/*lies $\}$ on the beach right now.

(b) Belgium $\{$ lies/*is lying $\}$ between France and the Netherlands.

(c) A statue of Bill Clinton \{stands/is standing\} in the plaza.

(d) I \{am living/live $\}$ in Chicago.

For a person, a process such as lie on the beach is something construed as occurring in bounded episodes, whereas the position of a country is relatively fixed and stable. Using the progressive in (7b) would signal a perfective construal of lie, suggesting 


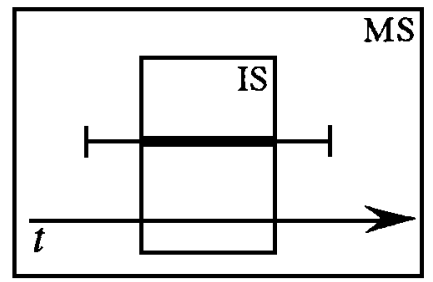

Figure 6. Progressive

that the location is only temporary - a possible construal but one unlikely in normal circumstances. With a statue, as in (7c), either construal is plausible. The progressive would suggest that the statue might be moved or replaced after the next election. Just from looking at the plaza, one could not determine whether a perfective or an imperfective construal of stand is appropriate - indeed, the absolute span of time may not be important (since all human circumstances are temporary). It is rather a matter of how the speaker chooses to construe and portray the situation. Similarly for (7d). Both versions might describe exactly the same objective situation, e.g. the speaker may have lived in Chicago for ten years and plan to live there just ten years more. The difference resides in whether living in the same city for twenty years is to be construed as a temporary residence or a permanent one, i.e. whether one focuses on the boundaries or abstracts away from them to emphasize the stability of the ongoing situation.

\section{The progressive and the tenses}

The English progressive construction with be. . -ing is only applicable to processes construed as perfective, hence temporally bounded. It is an imperfectivizing construction, so it does not apply to processes that are already imperfective (not bounded), where its effect would be vacuous (Langacker, 1987b).

Intuitively, we can describe the progressive in terms of 'zooming in' and taking an 'internal view' of a bounded event. Technically, I describe it as imposing an immediate temporal scope that excludes the endpoints of the perfective process it applies to. This is shown in figure 6. While the maximal scope is a span of time containing the full, bounded process, the immediate scope subtends just an arbitrary portion of its internal development. Only that portion is profiled, since - as a matter of definition - the profile is the focal point within the immediate scope. The overall progressive expression is imperfective, because grammatical class is determined by the profile and the profiled process is not bounded. Also, as with any imperfective, the profiled process is construed as being effectively homogeneous.

Because it only applies to perfectives, the progressive construction - though itself imperfective - signals that the original process is construed as being bounded. The subtle contrast between a basic imperfective and one derived by using the progressive 
(a)

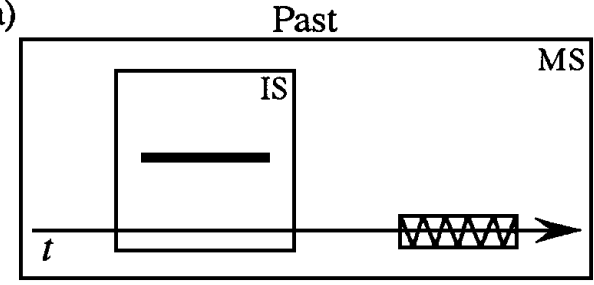

(b)

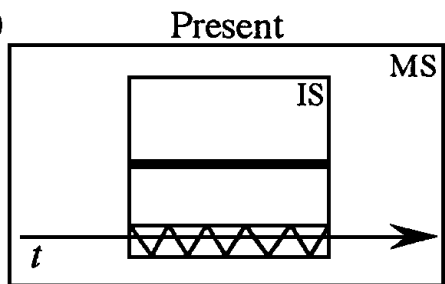

Figure 7. Tense

is seen by comparing figure 6 to figure $5(\mathrm{~b})$. Both profile a process which is unbounded within the immediate temporal scope (and construed as being effectively homogeneous). The difference is that a progressive expression creates this imperfective process by selectively attending to the interior of an overall occurrence recognized as being bounded. Thus in (7d) both the simple present and the progressive indicate a current residence in Chicago, but the latter portrays this as part of an overall residential episode of limited duration.

The progressive construction is purely aspectual: it does not itself make any specification concerning the location of the profiled process with respect to the time of speaking. The tense markers do relate the designated process to the time of speaking. English has just two tense markers, present and past. I analyze the future, will, as belonging to the modal system, which I cannot discuss here (see Langacker, 1991: 6.3).

I describe a tense marker as imposing an immediate temporal scope which is located with respect to the time of speaking (unlike the immediate scope imposed by the progressive). In particular, past tense specifies that this immediate scope is prior to the time of speaking, while present tense (at least in English) specifies that it precisely coincides with the time of speaking, as shown in figure 7. Note that the speech event is represented by a box with squiggly lines. In this particular diagram the heavy line is meant to be neutral in regard to whether the profiled process is perfective or imperfective.

It is required for both tenses that a full instantiation (or valid instance) of the process type in question occur within the immediate scope imposed. In the case of the present, whose essence is immediacy to the speech event, this process must further be coincident with the speech event (and hence the immediate scope). Succinctly stated, the past tense specifies that a full instance of the profiled process occurs prior to the time of speaking, and the present tense, that a full instance of the profiled process occurs and precisely coincides with the time of speaking. ${ }^{3}$

Both the progressive and the tenses impose an immediate temporal scope, thus delimiting what counts as the profiled process. They do so at different levels of

\footnotetext{
${ }^{3}$ Here I am only concerned with the temporal values of these elements, which are prototypical and cover most of their uses. To accommodate the full range of uses, I also posit more schematic meanings based on a notion of 'epistemic distance' (Langacker, 1991: 6.1).
} 


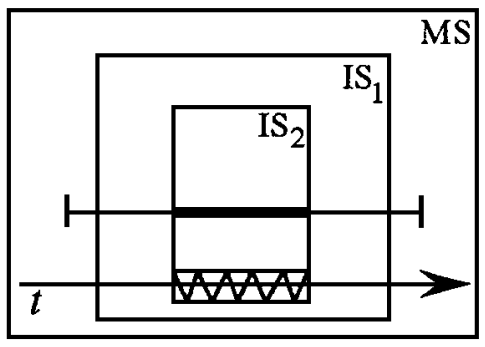

Figure 8. Present progressive

organization: the progressive applies to a verb stem at the first or lower level, while tense applies at a higher level to form a full, finite clause. Consequently, a progressive expression is itself marked for past or present tense. The progressive profiles an imperfective process - derived from a perfective verb by imposing a limited immediate scope - and tense marking then imposes its own immediate scope (figure 7). A tense-marked progressive thus has a maximal temporal scope and immediate scopes at two levels.

Consider first a present-tense progressive, e.g. They are working. In figure 8, $\mathrm{IS}_{1}$ represents the immediate scope imposed by the progressive construction, which falls within the boundaries of the perfective process designated by the verb. Within $\mathrm{IS}_{1}$, the present tense imposes another immediate scope, $I_{2}$, defined by the speech event. The profile of the overall expression is consequently that portion of the imperfective progressive process that coincides with the time of speaking.

In the case of the past progressive, there are two possible contextual interpretations. The immediate scope imposed by the tense marker, $\mathrm{IS}_{2}$, has to be prior to the speech event. However, nothing specifies where the speech event lies in relation to $\mathrm{IS}_{1}$, the immediate scope imposed by the progressive. In other words, the progressive process overall can either lie entirely prior to the speech event, or it can extend through the speech event. These possibilities are respectively illustrated in $(8 a-b)$ :

(8) (a) I was working this morning, but I am finished now.

(b) I was working this morning, and I still am.

They are diagrammed in figure 9 , representing the initial clauses in (8).

\section{The basic analysis}

Having presented the requisite background, together with the proposed characterization of the present tense, I can now proceed to showing how the analysis works in detail. How can it accommodate the actual facts of present-tense usage in English, which on first examination seem so problematic for a present-time meaning?

In fact, the analysis accounts for a wide range of data both straightforwardly and even rather elegantly. Let me start by showing the combination of past and present 
(a)

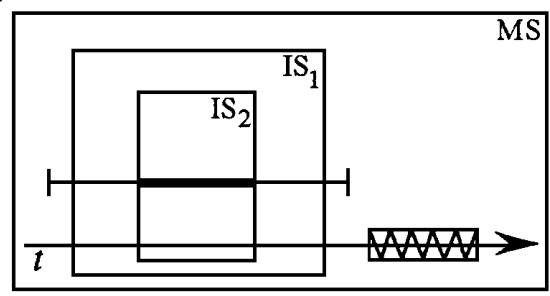

(b)

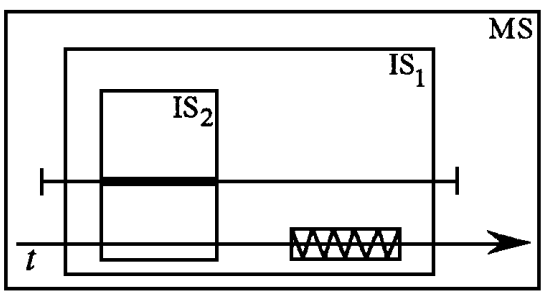

Figure 9. Past progressive

(a)

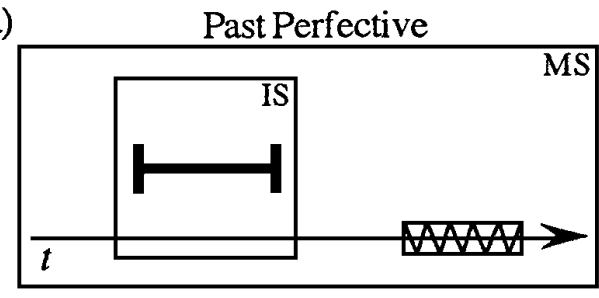

(c)

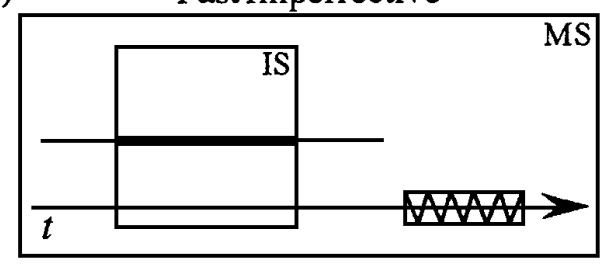

(b)

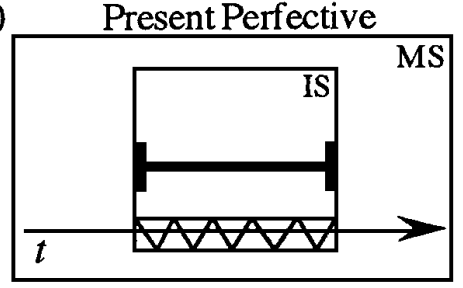

(d) Present Imperfective

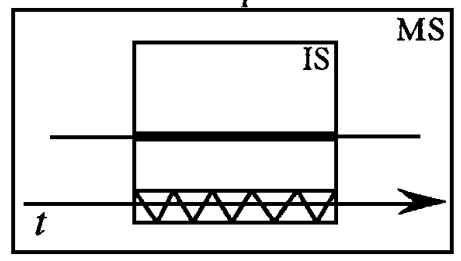

Figure 10. Tense-aspect combinations

tense with perfective and imperfective processes. The descriptions offered for these elements imply the configurations shown in figure 10.

Observe, first, that both perfectives and imperfectives freely occur in the past tense. This is so because the past tense makes no inherent specification concerning the size of the immediate scope it imposes for focused viewing. Thus, as seen in figure 10(a), it can always be made large enough to include a full instance of the profiled perfective process, regardless of its duration. As for imperfectives, recall that any subpart of an imperfective process is itself a valid instance of the category. So when the immediate scope delimits some portion of an overall imperfective process, as shown in figure $10(\mathrm{c})$, this portion - by definition constituting the processual profile - counts as a full instance occurring prior to the time of speaking, as the past tense requires.

Also accounted for is the felicity of imperfectives occurring in the present tense, sketched in figure 10(d). The reason is the same: the immediate scope, coincident with the time of speaking, delimits a portion of the overall imperfective process, 
thereby confining the processual profile to that segment. Owing to the mass-like nature of imperfectives, the profile constitutes a full instance of the imperfectiveprocess category, so the definition of the present tense is satisfied.

Next, since progressives are derived imperfectives, the analysis accounts for the occurrence of progressives in both the past and present tense. It can be seen that figure 8 is a special case of figure $10(\mathrm{~d})$, and the diagrams in figure 9 are special cases of figure $10(\mathrm{c})$.

The apparent problem of course lies with present-tense perfectives. There is nothing inherently anomalous about the configuration in figure 10(b); conceptually it is perfectly coherent. Why, then, are present-tense perfectives generally infelicitous, as in (9)?

(9) *Sarah \{learns a poem/makes a phone call/plants her garden $\}$ right now.

Present-tense perfectives are usually infelicitous, not because there is anything wrong with the situation in figure 10(b), but rather because - in normal circumstances - this situation is hard to achieve. Making the profiled event precisely coincide with the speech event poses two problems. The durational problem is that the length of an event is generally not equal to the length of a speech event describing it (i.e. the utterance of a finite clause which profiles it). It takes longer to plant a garden, for instance, than to utter the sentence Sarah plants her garden. The epistemic problem resides in having to observe an event and identify it prior to being able to report it. By the time the event is observed and identified, it is already too late to initiate a precisely coincident description. These problems do not arise with imperfectives. Given their mass-like character, with any portion of the overall process counting as an instance of the process type, the segment which coincides with the speech event will always qualify as an instance. So there is no durational problem. Nor is there an epistemic problem, since the profiled segment delimited by the speech event can follow a period of observation and still qualify as an instance.

The analysis further accounts for a striking exception to the nonoccurrence of present-tense perfectives, namely performatives, as in (10):

(10) (a) I order you to open that door.

(b) I promise that I will quit smoking.

(c) I hereby pronounce you man and wife.

Performatives are expressions which, when uttered under the proper circumstances, effect the very speech act they name (Austin, 1962; Searle, 1969). These are necessarily in the present tense, and despite their perfective nature, the sentences are completely felicitous. The reason is that, in the case of performatives, the speech event and the profiled process are one and the same, as a matter of definition. Since the process described and profiled by the main clause actually constitutes the act of speaking, as shown in figure 11, the durational problem does not arise. Nor does the epistemic problem, since the speaker can act and speak with prior intent. Because the speaker is not merely reporting on what happens but is rather in control of the event described, he does not have to observe the event in order to identify and describe it. 


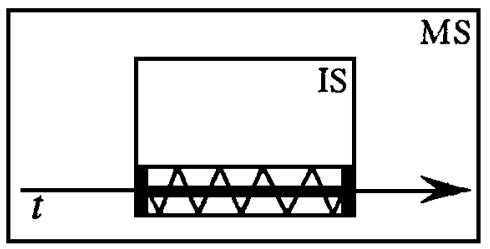

Figure 11. Performative

Hence there is no inherent conflict between bounding, implied by perfectivity, and temporal coincidence with the speech event, implied by the present tense. After all, the speech event is itself a bounded occurrence. The problem lies with the difficulty of achieving the configuration in figure 10(b) in particular kinds of circumstances, where the durational and epistemic problems arise. In particular, they arise in the default-case viewing arrangement, the one we normally assume unless there is reason to do otherwise. A presupposed viewing arrangement is one facet of construal, and figures in the overall meaning of every expression. In the default arrangement, the speaker and hearer are together in a fixed location, and report on actual occurrences they observe in the world around them. Departures from this canonical arrangement are of course many and frequent, yet it does appear to be basic.

In the default-case viewing arrangement, the durational and epistemic problems arise because the speaker merely reports on what happens, rather than controlling it, and since the reported event is independent of its description, affording no reason for them to have the same duration. The case of the speaker performing an action rather than simply describing it represents one kind of departure from the default, and in this circumstance the durational and epistemic problems are avoided, as we have seen.

Are there other kinds of departures from the default arrangement which have the same effect? It is easy to imagine some. We need only think of a situation where the speaker is in control of an event (to avoid the epistemic problem), and where its duration is approximately the right length, so that the speaker can make it coincide with a sentence describing it. A simple case is the concurrent description of bodily actions. Imagine the speaker carrying out the acts described in (11), coincident with each sentence:

(11) Now I raise my hand. Now I lower my hand. Now I stand up. Now I sit down.

The situation may seem artificial, but that is not important. The pertinent observation is that the present tense seems perfectly natural and appropriate in this circumstance, though each verb is perfective.

Another kind of example is given by the narration of a step-by-step demonstration. Imagine (12) being produced by the teacher in an origami class. In fact, it is easy to imagine an utterance being stretched out, with pauses, to achieve temporal coincidence (e.g. I make an incision . . from one corner . . to . . the . . center). 
(12) I pick up a sheet of paper and I fold it in two. I fold it again. Now I take the scissors, and I make an incision from one corner to the center.

What about the play-by-play mode of speech used by sportscasters? In a way, their role exemplifies the default viewing arrangement, since the announcer occupies a fixed position and does in fact report on actual occurrences observed from that position. How, then, is it possible to overcome the durational and epistemic problems, since present-tense perfectives are so common in this mode? Observe that the events described in this way (e.g. making a pass, taking a shot) have approximately the right duration for temporally coincident description. In the context of a sporting event, they are also quite stereotyped, hence the announcer has a good idea of what is likely to transpire at any instant. It is therefore possible to shadow the events fairly closely, sometimes even anticipating and describing them simultaneously with their occurrence. The goal at least is to come as close as possible to coincident description. The conventions of play-by-play reporting rest on either the fiction that this is feasible or else the tolerance of a certain time-lag.

I conclude, then, that the characterization of present tense based on temporal coincidence does in fact account for a wide array of data in a natural and straightforward way. But what about all the cases where the present tense is used for something other than present time? For these too a reasonable story can be told. Before I can tell it, however, I need to establish, on independent grounds, the linguistic prevalence and importance of 'virtual' or 'fictive' entities.

\section{The importance of virtual entities}

As described in the previous section, the default viewing arrangement involves the description of actual occurrences. Surprisingly often, however, normal language use involves the description of virtual or fictive entities, even when our real concern is with actual ones (Langacker, 1999b; Talmy, 1996). Here I can only provide a few illustrative examples, perhaps affording some idea of the broad variety of cases encountered.

It appears that fictivity is possible for any facet of an expression's meaning. For instance, the viewing arrangement itself can be virtual rather than actual. Sentence (13) presupposes the default viewing arrangement, where the speaker is stationary, and describes the scene from that perspective. However, this presupposed viewing arrangement is fictitious - in actuality, the speaker is in motion.

(13) The telephone poles are rushing past at ninety miles per hour.

The presupposed vantage point can also be a fictive one. In telling a story, for example, one can easily assume the spatial and temporal vantage point of a character. We see this in (14), where come and tomorrow adopt Jason's vantage point rather than the narrator's actual one.

(14) Jason was worried. The auditors were coming tomorrow. 
The speech act can also be fictitious. In posing a rhetorical question, for instance, the speaker only simulates an act of questioning, with no intention of the question being answered:

(15) Would I ever lie to you? I'm offended that you would even think of such a thing.

Many expressions describe fictive motion or fictive change (Langacker, 1986; Matsumoto, 1996a, 1996b, 1996c; Talmy, 1996). For example, a variety of motion verbs can appear in sentences describing static situations:

(16) The fence $\{$ goes/runs/stretches/reaches/extends\} from the front of his property all the way down to the river.

Observe that the situation being described is an actual one: there really is a fence with a certain extension. As is commonly the case, a virtual entity - in this case virtual motion - is invoked for purposes of describing an actual one.

An example of fictive change is (17):

(17) The mayor's limousine keeps getting longer.

In all likelihood, no actual limousine changes in length. The mayor's limousine is not interpreted as referring to any particular vehicle, but is rather a role description (Fauconnier, 1997), instantiated at different times by different vehicles. It is only by viewing these different instantiations of the role as if they were the same that a coherent conception of the profiled change emerges.

A role, such as the mayor's limousine, is one example of a thing that is virtual rather than actual. There are many other such examples. A virtual thing is often evoked for purposes of negation, as in (18a). The phrase a car does refer to an instance of the car category, but not any actual instance. ${ }^{4}$ Rather, it evokes a virtual instance, one 'conjured up' or imagined just for purposes of specifying the situation being denied.

(18) (a) Ellen doesn't have a car.

(b) Each boy was holding a frog.

Likewise in (18b), each boy does not refer to any particular boy, nor does a frog designate any actual frog. The boy and the frog referred to are fictive entities, conjured up to characterize a type of situation presented as being valid for each of the actual boys in some contextually identified group.

An entire profiled event and all its participants can be fictive, even in the description of an actual occurrence. Consider (19):

(19) Several times, a member of the audience interrupted with a question.

This sentence can perfectly well be used if there were, say, three interruptions, each involving a different member of the audience, as well as a different question. Yet the nouns are singular. This is because the finite clause does not profile an actual event, with actual participants, but rather a virtual event conjured up to represent the

${ }^{4}$ Observe that it makes no sense to ask Which particular car doesn't Ellen have?. 
abstract commonality of the three actual occurrences. It is used to make a local generalization about the kind of happening of which there were three instances.

The point of these examples is to make it clear that, when I talk about fictive entities in reference to the present tense, I am not inventing something just for that purpose. The invocation of fictive entities is something we easily and routinely do in normal language use, for myriad purposes.

\section{Nonpresent uses of the present}

Fictive entities are relevant for the characterization of nonpresent uses of the present tense. I suggest that in such uses what is being coded linguistically is not the actual occurrence of events, but rather their virtual occurrence as part of a noncanonical viewing arrangement. This virtual occurrence coincides with the time of speaking.

It is perhaps easiest to see this in the case of the scheduled future use of the present, as in (20a). I propose that such expressions relate only indirectly to the actual event in question. Rather, they evoke a kind of virtual schedule, a plan or projection concerning the expected occurrence and timing of future events. A virtual schedule consists of mental representations of anticipated events. Although one's ultimate concern is an actual event in the future, a sentence like (20a) directly describes a representation of this event. This is not inherently implausible. We do make plans and schedules. We frequently consult them. In reading from an actual schedule, we use the present tense, as in (20b). Moreover, I have already established that we commonly resort to the direct description of virtual entities, including virtual events, even when our ultimate concern is with actual ones (e.g. (19)).

(20) (a) Their plane arrives at noon.

(b) There it is on the monitor - their plane arrives at noon.

(c) ??Their plane arrives.

(d) ??An earthquake strikes next week.

Supporting the notion that something like a schedule is involved is the observation that the scheduled future strongly favors a time expression, as seen by the infelicity of (20c). Moreover, it does not work well for events that cannot be scheduled or anticipated. Thus (20d) is awkward unless it is uttered by God, or perhaps by a scientist with supreme confidence in a method of quake prediction.

The evocation of a virtual schedule is sketched in figure 12. The schedule consists of virtual events, which are representations of anticipated actual events. Dotted correspondence lines indicate that the time interval through which each virtual event is conceived as unfolding is identified with a particular time in the future. However, the events constituting the schedule are only virtual. Although it pertains to the future, the schedule itself is stable and mentally accessible through a span of time that includes the present.

Metaphorically, it is helpful to think of a virtual schedule as a 'document' available to be 'read' at any time. In producing a sentence like (20a), the speaker is 


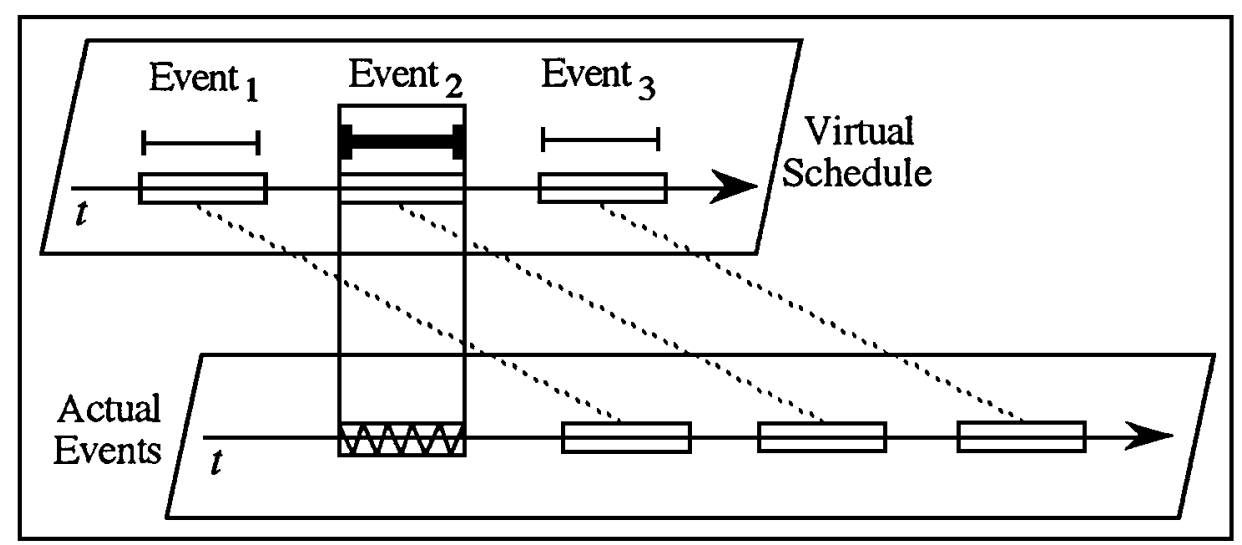

Figure 12. Scheduled future

essentially reading off one of its entries. In the context of consulting a virtual schedule, what counts as the occurrence of a process is the virtual occurrence consisting of an entry being read. When this reading resides in the production of a finite clause describing it, the reading - and hence the event's occurrence - is coincident with the speech event. Use of the present tense thus conforms to the proposed definition, taking into account the presupposed viewing arrangement.

I should emphasize that every expression invokes a presupposed viewing arrangement as part of the conceptual substrate that supports its meaning and shapes its form. In the default-case viewing arrangement, described earlier, we tend to be unaware of this precisely because it constitutes the default. Yet there are many kinds of departures from the default. Furthermore, we have already seen how the viewing arrangement affects the use of present tense. ${ }^{5}$ What it means for an event to 'occur' has to be interpreted relative to the viewing arrangement involved. The canonical arrangement, where the speaker observes and reports the occurrence of actual events, can be seen as a special, albeit privileged, circumstance. In a noncanonical arrangement where the speaker is consulting a virtual document, the events are virtual and they occur in the sense of being read.

As a general matter, the key to understanding 'nonpresent' uses of the present tense is to recognize the special viewing arrangements they presuppose. They all diverge from the default arrangement by invoking some kind of mental construction - such as a schedule - consisting of event representations. While these may correspond in some fashion to actual events, it is the represented events that are directly coded linguistically and profiled by the present-tense verb. What counts as the occurrence of such a process is therefore not an actual occurrence, but rather a

5 Recall, for instance, that the default arrangement is actually responsible for the durational and epistemic problems posed by present-tense perfectives. 
virtual one consisting in its apprehension in the manner indicated by the special viewing arrangement (e.g. reading the virtual schedule).

Details naturally vary. A number of cases are reasonably described metaphorically as the reading of a virtual document, differing in the kind of document envisaged. Consider stage directions, as in (21):

(21) Hamlet moves to center stage. He pulls out his dagger. He examines it.

Here the script may well be physically embodied, and read from literally. Even conceived as a virtual document, it comprises a series of inscribed events available for 'reading' at any time. Reading them - apprehending the successive event descriptions - induces their virtual occurrence in the form of mentally constructing the prescribed event sequence.

Stage directions are comparable in this respect to such documents as recipes, directions, and instruction manuals, which sometimes employ the present tense (another option being imperatives):

(22) You head north on highway 107. You take the westbound exit onto Hillcrest Drive. You go about 3 miles until you come to a traffic light. There you turn left. You continue for another mile...

The successive events described are not actual, but virtual ones involving a virtual actor (the impersonal you), awaiting actualization by anybody who chooses to follow the instructions. Although the instructions may be physically written down, and may actually be carried out, the document per se is a mental construction comprising virtual events whose occurrence - marked by present tense - is a virtual occurrence consisting in the instructions being read and apprehended.

The narration of a demonstration can also be viewed in these terms. Imagine a cooking demonstration on TV. In one possible style of narration, each present-tense utterance precedes the action it describes rather than coinciding with it.

(23) First I take six eggs . . I crack them and empty them into a mixing bowl . . Now I measure out two cups of flour . . I put them in the bowl with the eggs . . . Next I beat the mixture until it is well blended . . .

While the events in question are correlated with actions, I suggest that they are being conceptualized more abstractly as entries in a list, collectively constituting a kind of script or scenario that is being followed. The present-tense verbs are not in fact being used for the direct description of actual events; instead they indicate the reading off of entries on this virtual document. In this noncanonical viewing arrangement, the events' virtual occurrence does coincide with the time of speaking.

Consider next the historical present:

(24) I'm driving home last night and I hear a siren. I pull over and stop. This cop comes up and starts writing me a ticket.

Here the virtual document consists of a series of recalled events. Or perhaps we should use the metaphor of a video tape, which the speaker can mentally 'replay' at leisure, at the pace required for coincident linguistic encoding. 
A different viewing arrangement involving recall is the reading of a photo caption, e.g. (25). The statement of course is physically instantiated and is read quite literally. The photo captures one moment of the event described by the caption. It helps to evoke the event's virtual occurrence, consisting in the reader's apprehension of the statement's import.

(25) Nixon says farewell from the steps of his helicopter.

An important class of mental constructions consists of generalizations extracted to represent the world's basic structure, as opposed to specific, contingent occurrences that arise within this stable framework (Goldsmith \& Woisetschlaeger, 1982; Langacker, 1996, 1997). Expressions describing the world's basic structure - which I refer to as structural statements - include generics, habituals, and 'timeless truths', respectively exemplified in $(26 a-c)$.

(26) (a) A kitten is born blind.

(b) I drink my whisky on the rocks.

(c) The earth revolves around the sun.

Like example (19), these statements profile virtual events conjured up to capture a generalization. But in contrast to (19), which describes the commonality inherent in a set of contingent occurrences, the generalizations in (26) are inscribed on a virtual document representing stable facets of the world's basic nature. The present-tense verbs of course do not refer to specific, actual events, e.g. (26a) does not designate any actual instance of a kitten being born. The profiled events are virtual instances of the process types in question, conjured up to express generalizations about the world's structure. They are mental constructions, each corresponding to an openended set of actual instantiations, distributed throughout the time span during which the generalization holds. The tense marking specifies the time at which the event representation can be consulted as a way of apprehending this facet of the world's structure. Present tense indicates its viewing (hence its virtual occurrence) coincident with the time of speaking.

Last, we need to consider the use of present tense in certain types of subordinate clauses, as in (27):

(27) $\{$ If/when/until/before/after/while $\}$ you sell your house, you should think about retiring.

Despite its present-tense form, the verb sell refers to a process envisaged as occurring in the future. Here I can do no better than follow the analysis proposed by Fauconnier (1997) in terms of mental spaces. The subordinators introducing these clauses are what he calls space builders: if establishes a hypothetical space, and the others set up spaces defined by their temporal location. They further shift the viewpoint to the space they establish. They thus invoke a special viewing arrangement in which the clausal content is apprehended from a fictive temporal vantage point, rather than the actual time of speaking. Mental transfer to a fictive vantage point is of course a well-attested phenomenon, previously illustrated in (14). Given 
this mental transfer, such that the time of speaking is fictively located inside the mental space, a process conceived as occurring in this space is fictively viewed as coincident with the speech event.

\section{Conclusion}

This analysis of the English present tense is quite nonstandard and undoubtedly controversial. It is still preliminary, and certainly incomplete. ${ }^{6}$ Even so, I have little personal doubt that the account is basically valid, or at least headed in the right direction. Once explained, it is actually rather simple and straightforward - what could be more straightforward than the present tense meaning "coincident with the time of speaking? When this characterization is combined with other notions, all independently proposed and justified, a wide array of seemingly recalcitrant data falls into place quite naturally.

But if it is simple and natural, why has it not been obvious all along? Why does virtually every analyst deny that the present tense is an indication of present time? Let me suggest that traditional ways of looking at tense engender confusion by obscuring its basic nature. Traditional semantics is objectivist in spirit, ignoring construal and the subjective basis of factors like bounding. In describing tense, it is usual to focus exclusively on the temporal relation between the actual time of speaking and the full duration of an actual event occurrence. No attention is paid to fictivity or the myriad viewing arrangements which mediate between objective circumstances and the formulation of linguistic expressions. In short, traditional assumptions leave out essentially everything needed for a viable account. Thus a cogent description remains elusive, and the present tense is claimed to be anything but a present tense.

I hope to have made it evident that general principles of cognitive semantics make possible a description that is natural, insightful, and empirically adequate. I am not unhappy with the conclusion that the so-called present tense does indeed deserve to be so called.

Author's address:

Department of Linguistics, 0108

University of California, San Diego

La Jolla, CA 92093-0108

USA

rwl@ling.ucsd.edu

${ }^{6}$ For instance, I have not sufficiently emphasized epistemic immediacy, which I see as the flip side of coincidence with the time of speaking (Brisard, 1999). Observe that the reading of a virtual document can either be described as involving immediacy in the apprehension of an event or else temporal coincidence of its virtual occurrence. 


\section{References}

Austin, J. L. (1962). How to do things with words. Cambridge, MA: Harvard University Press.

Brisard, F. (1999). A critique of localism in and about tense theory. Antwerp: University of Antwerp doctoral dissertation.

Fauconnier, G. (1997). Mappings in thought and language. Cambridge: Cambridge University Press.

Goldsmith, J. \& E. Woisetschlaeger (1982). The logic of the English progressive. Linguistic Inquiry 13: 79-89.

Langacker, R. W. (1986). Abstract motion. Proceedings of the Annual Meeting of the Berkeley Linguistics Society 12: 455-71.

Langacker, R. W. (1987a). Foundations of cognitive grammar, vol. 1, Theoretical prerequisites. Stanford: Stanford University Press.

Langacker, R. W. (1987b). Nouns and verbs. Language 63: 53-94.

Langacker, R. W. (1990). Concept, image, and symbol: the cognitive basis of grammar. Berlin and New York: Mouton de Gruyter.

Langacker, R. W. (1991). Foundations of cognitive grammar, vol. 2, Descriptive application. Stanford: Stanford University Press.

Langacker, R. W. (1993). Grammatical traces of some 'invisible' semantic constructs. Language Sciences 15: 323-55.

Langacker, R. W. (1996). A constraint on progressive generics. In Goldberg, A. E. (ed.), Conceptual structure, discourse and language. 289-302. Stanford: CSLI Publications.

Langacker, R. W. (1997). Generics and habituals. In Athanasiadou, A. \& R. Dirven (eds.), On conditionals again. 191-222. Amsterdam and Philadelphia: John Benjamins.

Langacker, R. W. (1999a). Grammar and conceptualization. Berlin and New York: Mouton de Gruyter.

Langacker, R. W. (1999b). Virtual reality. Studies in the Linguistic Sciences 29.2: 77-103.

Matsumoto, Y. (1996a). How abstract is subjective motion? A comparison of coverage path expressions and access path expressions. In Goldberg, A. E. (ed.), Conceptual structure, discourse and language. 359-73. Stanford: CSLI Publications.

Matsumoto, Y. (1996b). Subjective motion and English and Japanese verbs. Cognitive Linguistics 7: 183-226.

Matsumoto, Y. (1996c). Subjective-change expressions in Japanese and their cognitive and linguistic bases. In Fauconnier, G. \& E. Sweetser (eds.), Spaces, worlds, and grammar. 124-56. Chicago and London: University of Chicago Press.

Searle, J. R. (1969). Speech acts: an essay in the philosophy of language. London and New York: Cambridge University Press.

Talmy, L. (1996). Fictive motion in language and 'ception'. In Bloom, P., M. A. Peterson, L. Nadel, \& M. F. Garrett (eds.), Language and space. 211-76. Cambridge, MA, and London: MIT Press/Bradford. 